\title{
MAXIMAL REGULARITY OF DISCRETE SECOND ORDER CAUCHY PROBLEMS IN BANACH SPACES
}

\author{
CLAUDIO CUEVAS AND CARLOS LIZAMA
}

\begin{abstract}
We characterize the discrete maximal regularity for second order difference equations by means of spectral and $R$-boundedness properties of the resolvent set.
\end{abstract}

\section{INTRODUCTION}

Let $X$ be a Banach space and let $A$ be a bounded linear operator. For a given sequence $\left(f_{n}\right)_{n \in \mathbb{Z}_{+}}$in $X$, the problem of producing a sequence $\left(u_{n}\right)_{n \in \mathbb{Z}_{+}}$such that

$$
\Delta^{2} u_{n}-A u_{n}=f_{n},
$$

for all $n \in \mathbb{Z}_{+}$with the initial conditions $u_{0}=0$ and $u_{1}=0$ is called a discrete second order Cauchy problem.

Consider a space $Y(X)$ of $X$-valued sequences. If $\left(f_{n}\right)_{n \in \mathbb{Z}_{+}} \in Y(X)$ implies always $\left(\Delta^{2} u_{n}\right)_{n \in \mathbb{Z}_{+}} \in Y(X)$, then the Cauchy problem is said to have $Y$-discrete maximal regularity.

Maximal regularity of continuous time evolution equations has received much attention in recent years. Concerning discrete time, $l_{p^{-}}$discrete maximal regularity for the evolution equation $\Delta u_{n}-A u_{n}=f_{n}$ with initial condition $u_{0}=0$ has been studied by the first time by Blunck [5], [6] and Portal [17]. Recently, discrete maximal regularity for functional difference equations with infinite delay was considered by Cuevas-Vidal [11].

Maximal regularity properties for the vector-valued discrete time equation (1.1) appears not to be considered in the literature. An obvious analogy with the continuous case suggests a wide range of problems which deserve to be investigated.

A motivation for this studies stems in the recent article by Palencia and Piskarev [16] where the authors shown that the Cauchy problem for second order equations $u^{\prime \prime}(t)=$ $A u(t)+f(t)$ has the maximal regularity property in $L^{p}([0, T] ; X)$ for some $p \in[1, \infty]$ and $T>0$ if, and only if, $A$ is bounded.

We observe that the study of maximal regularity is very useful for treating semilinear and quasilinear problems. Results in this direction have been studied extensively in recent years (see for example Amann [1], Denk-Hieber and Prüss [12], Clément-Londen-Simonett [10], the survey by Arendt [2] and the bibliography therein). One of the most important modern tools to prove maximal regularity are operator-valued Fourier multiplier theorems.

2000 Mathematics Subject Classification. Primary 39A12 ; Secondary 42A45, 47D09.

Key words and phrases. Discrete maximal regularity; Second order difference equations; $R$-boundedness; UMD spaces.

The first author is partially supported by CNPQ/Brazil Grant\# 300068/2005-0.

The second author is partially supported by Proyecto Anillo ACT-13. 
Given $T \in \mathcal{B}(X)$, in this paper we are able to characterize the discrete maximal regularity of (1.1) by $R$-boundedness properties of the resolvent operator of $I-T$, namely we will prove that the following assertions are equivalent in a $U M D$ space $X$ :

(i) $T$ has discrete maximal regularity of order 2 .

(ii) $\left\{(z-1)^{2} R\left((z-1)^{2}, I-T\right):|z|=1, z \neq 1\right\}$ is $R$-bounded.

To obtain this characterization, we use a recent result due to Blunck (see Theorem 1.3 of $[5])$.

We remark that the concept of $R$-boundedness was introduced by Bourgain [7] and play a fundamental role in recent works by Clément-Da Prato [8], Clément et al. [9], Weis $[18,19]$, Arendt-Bu [3, 4] and Keyantuo-Lizama [13, 14, 15].

For a quick overview of the organization of the paper, we present in Section 2 an explanation for the the basic notations and we introduce the definition of the discrete maximal regularity for the second order difference equations. To facilitate a comprehensive understanding to the reader we have supplied several basic $R$-boundedness properties which are a natural tool in our setting. We show how they can be used to obtain our characterization about maximal regularity for equation (1.1). We also treat in Section 3 the same problem from the point of view of first order reduction.

\section{Discrete maXimal Regularity FOR the SECOND ORDER PROBlem}

Let $X$ be a Banach space. Let $\mathbb{Z}_{+}$denote the set of positive integer numbers, $\Delta$ the difference operator of the first order, i.e. for each $x: \mathbb{Z}_{+} \rightarrow X$, and $n \in \mathbb{Z}_{+}, \Delta x_{n}=$ $x_{n+1}-x_{n}$.

For $T \in \mathcal{B}(X)$ be given, define $\mathcal{T}: \mathbb{Z}_{+} \rightarrow \mathcal{B}(X)$ by $\mathcal{T}(n)=T^{n}$ and consider the discrete time evolution equation

$$
\Delta x_{n}-(T-I) x_{n}=f_{n}, \quad x_{0}=x
$$

or, equivalently,

$$
x_{n+1}-T x_{n}=f_{n} \text { for all } n \in \mathbb{Z}_{+}, \quad x_{0}=x,
$$

where the sequence $f=\left(f_{n}\right)$ is given. Then the (unique) solution $x$ is given by $x_{n+1}=$ $\mathcal{T}(n) x+(\mathcal{T} * f)_{n}$ where

$$
(\mathcal{T} * f)_{n}:=\sum_{k=0}^{n} \mathcal{T}(k) f_{n-k}=\sum_{k=0}^{n} T^{k} f_{n-k}, \quad n \in \mathbb{Z}_{+} .
$$

Let $\left(f_{n}\right) \in l_{p}\left(\mathbb{Z}_{+} ; X\right)$ and denote by $x$ the solution of the equation

$$
x_{n+1}-T x_{n}=f_{n} \text { for all } n \in \mathbb{Z}_{+}, \quad x_{0}=0 .
$$

Then

$$
\Delta x_{n}=\sum_{k=0}^{n}(T-I) T^{k} f_{n-k}=:\left(\mathcal{T}^{\prime} * f\right)_{n} .
$$

Recall that $T \in \mathcal{B}(X)$ is said to be power bounded if $\sup _{n \in \mathbb{Z}_{+}}\left\|T^{n}\right\|<\infty$ or, equivalently, $\mathcal{T} \in l_{\infty}\left(\mathbb{Z}_{+}, X\right)$. The following definition was introduced by Blunck [6, p.212].

Definition 2.1. Let $1<p<+\infty$. Let $T \in \mathcal{B}(X)$ be a power bounded operator. We say that $T$ has discrete maximal regularity of order 1 if $\mathcal{K}_{1} f:=\mathcal{T}^{\prime} * f$ defines a bounded operator $\mathcal{K}_{1} \in \mathcal{B}\left(l_{p}\left(\mathbb{Z}_{+}, X\right)\right)$. 
This property of $T \in \mathcal{B}(X)$ has been proved to be independent of $p \in(0, \infty)$.

In this section, we consider the equation

$$
\Delta^{2} x_{n}-(I-T) x_{n}=f_{n} \text { for all } n \in \mathbb{Z}_{+}, \quad x_{0}=x, \quad \Delta x_{0}=x_{1}-x_{0}=y .
$$

where $T \in \mathcal{B}(X), \Delta^{2} x_{n}=\Delta\left(\Delta x_{n}\right)$ and $f: \mathbb{Z}_{+} \rightarrow X$.

Denote $\mathcal{C}(0)=I$, the identity operator on $X$, and define

$$
\mathcal{C}(n)=\sum_{k=0}^{[n / 2]}\left(\begin{array}{c}
n \\
2 k
\end{array}\right)(I-T)^{k} \quad \text { for } n=1,2, \ldots
$$

and $\mathcal{C}(n)=\mathcal{C}(-n)$ for $n=-1,-2, \ldots$ We define also $\mathcal{S}(0)=0$,

$$
\mathcal{S}(n)=\sum_{k=0}^{[(n-1) / 2]}\left(\begin{array}{c}
n \\
2 k+1
\end{array}\right)(I-T)^{k}
$$

for $n=1,2, \ldots$ and $\mathcal{S}(n)=-\mathcal{S}(-n)$ for $n=-1,-2, \ldots$

Considering the above notations we can now state the following result.

Proposition 2.2. Let $T \in \mathcal{B}(X)$ be given, then the (unique) solution of equation (2.6) is given by

$$
x_{m+1}=\mathcal{C}(m) x+\mathcal{S}(m) y+(\mathcal{S} * f)_{m} .
$$

Moreover,

$$
\Delta x_{m+1}=(I-T) \mathcal{S}(m) x+\mathcal{C}(m) y+(\mathcal{C} * f)_{m} .
$$

Proof. Observe that equation (2.6) is equivalent to the following:

$$
x_{n+2}-2 x_{n+1}+T x_{n}=f_{n} \text { for all } n \in \mathbb{Z}_{+}, x_{0}=x, \Delta x_{0}=y .
$$

Let $v_{n}:=\left[x_{n}, \Delta x_{n}\right], F_{n}:=\left[0, f_{n}\right]$ and $R_{T} \in \mathcal{B}(X \times X)$ defined by

$$
R_{T}[x, y]=[x+y, x-T x+y] .
$$

Then it is not difficult to see that equation (2.6) is equivalent to:

$$
v_{n+1}-R_{T} v_{n}=F_{n}, \quad v_{0}=\left(x_{0}, \Delta x_{0}\right)=(x, y) .
$$

which has the solution (see also (2.3))

$$
v_{m+1}=R_{T}^{m} v_{0}+\sum_{n=0}^{m} R_{T}^{n} F_{m-n} .
$$

Denote

Then a calculation show us that

$$
R_{T}=\left[\begin{array}{cc}
I & I \\
I-T & I
\end{array}\right]
$$

$$
R_{T}^{n}=\left[\begin{array}{cc}
\mathcal{C}(n) & \mathcal{S}(n) \\
(I-T) \mathcal{S}(n) & \mathcal{C}(n)
\end{array}\right]
$$


The result is now a consequence of formula (2.13).

Remark 2.3. Let $T \in \mathcal{B}(X)$ be given and suppose that $(I-T)^{1 / 2}$ exists. Then

$$
\mathcal{C}(n)=\frac{\left(I+(I-T)^{1 / 2}\right)^{n}+\left(I-(I-T)^{1 / 2}\right)^{n}}{2}
$$

and

$$
(I-T)^{1 / 2} \mathcal{S}(n)=\frac{\left(I+(I-T)^{1 / 2}\right)^{n}-\left(I-(I-T)^{1 / 2}\right)^{n}}{2}
$$

Define $P_{T}:=I+(I-T)^{1 / 2}$ and $N_{T}:=I-(I-T)^{1 / 2}$. If $P_{T}$ and $N_{T}$ are power bounded then $T$ is power bounded. The converse is in general not true.

Now, let $B$ and $P$ be the operators in $\mathcal{B}(X \times X)$ defined by

$$
\begin{aligned}
B[x, y] & =\left[N_{T} x, P_{T} y\right], \\
P[x, y] & =\left[\frac{1}{2}(I-T)^{-1 / 2}(y-x), \frac{1}{2}(x+y)\right] .
\end{aligned}
$$

Note that $P$ is invertible and

$$
P^{-1}[x, y]=\left[-(I-T)^{1 / 2} x+y,(I-T)^{1 / 2} x+y\right] .
$$

Is not difficult to see that $R_{T}=P B P^{-1}$ and that the solution of equation (2.11) can be written as

$$
x_{m+1}=\frac{1}{2}\left(P_{T}^{k}+N_{T}^{k}\right) x+(I-T)^{-1 / 2} \frac{1}{2}\left(P_{T}^{k}-N_{T}^{k}\right) y+\sum_{k=0}^{n}(I-T)^{-1 / 2} \frac{1}{2}\left(P_{T}^{k}-N_{T}^{k}\right) f_{n-k} .
$$

The following definition is the natural extension of the concept of maximal regularity for the continuous case; cf. [16].

Definition 2.4. Let $1<p<+\infty$. We say that $T \in \mathcal{B}(X)$ has discrete maximal regularity of order 2 if $\mathcal{K}_{2} f:=\sum_{k=1}^{n}(I-T) \mathcal{S}(k) f_{n-k}$ defines a bounded operator $\mathcal{K}_{2} \in \mathcal{B}\left(l_{p}\left(\mathbb{Z}_{+}, X\right)\right)$.

As consequence of the definition, if $T \in \mathcal{B}(X)$ has discrete maximal regularity of order 2 then $T$ has discrete $l_{p}$-maximal regularity, that is, for each $\left(f_{n}\right) \in l_{p}\left(\mathbb{Z}_{+} ; X\right)$ we have $\left(\Delta^{2} x_{n}\right) \in l_{p}\left(\mathbb{Z}_{+} ; X\right)$, where $\left(x_{n}\right)$ is the solution of the equation

$$
\Delta^{2} x_{n}-(I-T) x_{n}=f_{n} \text { for all } n \in \mathbb{Z}_{+}, \quad x_{0}=0, \quad x_{1}=0 .
$$

Moreover,

$$
\Delta^{2} x_{n}=\sum_{k=1}^{n}(I-T) \mathcal{S}(k) f_{n-k}
$$

Next, we will recall the concept of $R$-boundedness. For $j \in \mathbb{N}$, denote by $r_{j}$ the $j$-th Rademacher function on $[0,1]$, i.e. $r_{j}(t)=\operatorname{sgn}\left(\sin \left(2^{j} \pi t\right)\right)$. For $x \in X$ we denote by $r_{j} \otimes x$ the vector valued function $t \longrightarrow r_{j}(t) x$. 
Definition 2.5. A family $\mathbf{T} \subset \mathcal{B}(X)$ is called $R$-bounded if there exists $C_{q} \geq 0$ such that:

$$
\left\|\sum_{j=1}^{n} r_{j} \otimes T_{j} x_{j}\right\|_{L^{q}(0,1, X)} \leq C_{q}\left\|\sum_{j=1}^{n} r_{j} \otimes x_{j}\right\|_{L^{q}(0,1, X)}
$$

for all $T_{1}, \ldots, T_{n} \in \mathbf{T}, x_{1}, \ldots, x_{n} \in X$ and $n \in \mathbb{N}$, where $1 \leq q<\infty$. We denote by $R_{q}(T)$ the smallest constant $C_{q}$ such that (2.17) holds.

It is clear from the definition that any $R$-bounded family is bounded. The converse of this assertion holds only in spaces which are isomorphic to Hilbert spaces. For more details, we refer to Arendt-Bu [3, 4].

In order to prove the next theorem we need some basic properties of $R$-boundedness.

Remark 2.6. a) Let $\mathbf{S}, \mathbf{T} \subseteq \mathcal{B}(X)$ be $R$-bounded sets, then $\mathbf{S} \cdot \mathbf{T}:=\{S \cdot T: S \in \mathbf{S}, T \in \mathbf{T}\}$ and $\mathbf{S} \pm \mathbf{T}:=\{S \pm T: S \in \mathbf{S}, T \in \mathbf{T}\}$ are $R$-bounded and $R_{q}(\mathbf{S} \cdot \mathbf{T}) \leq R_{q}(\mathbf{S}) R_{q}(\mathbf{T})$ and $R_{q}(\mathbf{S} \pm \mathbf{T}) \leq R_{q}(\mathbf{S})+R_{q}(\mathbf{T})$. Further, $R_{q}(\{T\}) \leq\|T\|$.

b) Each subset $M \subseteq \mathcal{B}(X)$ of the form $M=\{\lambda I: \lambda \in \Omega\}$ is $R$-bounded whenever $\Omega \subseteq \mathbb{C}$ is bounded ( $I$ denotes the identity operator on $X$ ). This follows from Kahane's inequality (see [1, Lemma 1.7]).

Given $f \in l_{p}(\mathbb{Z} ; X)$ we recall that the Fourier transform on $\mathbb{T}:=\{z \in \mathbb{C}:|z|=1\}$ is defined as

$$
\mathcal{F} f(z)=\hat{f}(z)=\sum_{j \in \mathbb{Z}} z^{-j} f(j), \quad z \in \mathbb{T} .
$$

Proposition 2.7. Let $T \in \mathcal{B}(X)$ and suppose $\left\{(z-1)^{2}\right\}_{z \in \mathbb{T}} \subseteq \rho(I-T)$. Then

$$
\hat{\mathcal{S}}(z)=z\left((z-1)^{2}-(I-T)\right)^{-1}, \quad z \in \mathbb{T},
$$

and

$$
\hat{\mathcal{C}}(z)=z(z-1)\left((z-1)^{2}-(I-T)\right)^{-1}, \quad z \in \mathbb{T} .
$$

Proof. Given $x \in X$ we define

$$
f_{n}= \begin{cases}x & \text { for } n=0 \\ 0 & \text { for } n \neq 0 .\end{cases}
$$

A direct calculation shows that $\hat{f}(z)=x$ for $z \in \mathbb{T}$. Consider the problem

$$
\Delta^{2} x_{n}-(I-T) x_{n}=f_{n} \text { for all } n \in \mathbb{Z}_{+}, \quad x_{0}=0, \quad \Delta x_{0}=0 .
$$

By Proposition 2.2 the (unique) solution is given by $x_{n+1}=(\mathcal{S} * f)(n)$. Then $z \hat{x}(z)=$ $\widehat{\mathcal{S}}(z) \hat{f}(z)=\hat{\mathcal{S}}(z) x, z \in \mathbb{T}$. On the other hand, note that a direct calculation gives $\widehat{\Delta x}(z)=$ $(z-1) \hat{x}(z)$ for $z \in \mathbb{T}$. Hence, applying Fourier transform in (2.20) and then multiplying the result by $z$, we obtain

$$
z x=\left((z-1)^{2}-(I-T)\right) z \hat{x}(z)=\left((z-1)^{2}-(I-T)\right) \hat{\mathcal{S}}(z) x, \quad z \in \mathbb{T},
$$

obtaining the first assertion. To prove the second one, we note that by Proposition 2.2 we have $\Delta x_{n+1}=(\mathcal{C} * f)(n)$ and then $z(z-1) \hat{x}(z)=z \widehat{\Delta x}(z)=\hat{\mathcal{C}}(z) x, z \in \mathbb{T}$. Therefore, applying Fourier transform in (2.20) and then multiplying the result by $z(z-1)$ we get

$$
z(z-1) x=\left((z-1)^{2}-(I-T)\right) z(z-1) \hat{x}(z)=\left((z-1)^{2}-(I-T)\right) \hat{\mathcal{C}}(z) x, \quad z \in \mathbb{T},
$$


and the proof is finished.

We recall now the following operator-valued multiplier theorem on $\mathbb{T}$ due to Blunck $[5$, Theorem 1.3].

Theorem 2.8. Let $p \in(0, \infty)$ and $X$ be a $U M D$ space. Let $M:(-\pi, \pi) \backslash\{0\} \rightarrow \mathcal{B}(X)$ be a differentiable function such that the set

$$
\left\{M(t),\left(e^{i t}-1\right)\left(e^{i t}+1\right) M^{\prime}(t): t \in I\right\}
$$

is $R$-bounded. Then there is an operator $T_{M} \in \mathcal{B}\left(l_{p}(\mathbb{Z} ; X)\right)$ such that

$$
\mathcal{F}\left(T_{M} f\right)\left(e^{i t}\right)=M(t) \mathcal{F} f\left(e^{i t}\right), \quad t \in I, \mathcal{F} f \in L_{\infty}(\mathbb{T} ; X)
$$

of compact support.

Definition 2.9. An operator $S \in \mathcal{B}(X)$ is called analytic if the set

$$
\left\{n(S-I) S^{n}: n \in \mathbb{N}\right\}
$$

is bounded.

In what follows, we denote $\mathbb{D}(z, r)=\{w \in \mathbb{C}:|w-z|<r\}$.

Lemma 2.10. Let $T \in \mathcal{B}(X)$ such that $T$ is analytic. Then $\sigma(I-T) \subseteq \mathbb{D}(1,1) \cup\{0\}$. In particular, $(z-1)^{2} \in \rho(I-T)$ whenever $|z|=1, z \neq 1$.

Proof. Let $M>0$ such that $\frac{M}{n} \geq\left\|T^{n}(T-I)\right\|$ for all $n \in \mathbb{N}$. Define $p(z)=z^{n+1}-z^{n}$. By the spectral mapping theorem, we have

$$
\begin{aligned}
\left\|T^{n}(T-I)\right\| & \geq \sup _{\lambda \in \sigma(p(T))}|\lambda|=\sup _{\lambda \in p(\sigma(T))}|\lambda| \\
& =\sup _{z \in \sigma(T)}\left|z^{n}(z-1)\right|=\sup _{w \in \sigma(I-T)}\left|w(1-w)^{n}\right| \geq|w||1-w|^{n},
\end{aligned}
$$

for all $w \in \sigma(I-T), n \in \mathbb{N}$. Hence $\sigma(I-T) \subseteq \mathbb{D}(1,1) \cup\{0\}$. For the last assertion we note that $\left|\left(e^{i \theta}-1\right)^{2}-1\right|^{2} \geq 1$ for all $\theta \in(0,2 \pi)$.

The following is the main result of this paper.

Theorem 2.11. Let $X$ be a $U M D$ space and let $T \in \mathcal{B}(X)$ be power bounded and such that $T$ is analytic. Then the following assertions are equivalent.

(i) $T$ has discrete maximal regularity of order 2.

(ii) $\left\{(\lambda-1)^{2} R\left((\lambda-1)^{2}, I-T\right):|\lambda|=1, \lambda \neq 1\right\}$ is R-bounded.

Proof. $\quad(i) \Rightarrow($ ii $)$ Define $k_{T}: \mathbb{Z} \rightarrow \mathcal{B}(X)$ by

$$
k_{T}(n)= \begin{cases}(I-T) \mathcal{S}(n) & \text { for } n \in \mathbb{N} \\ 0 & \text { otherwise }\end{cases}
$$

and the corresponding operator $K_{T}: l_{p}(\mathbb{N} ; X) \rightarrow l_{p}(\mathbb{N} ; X)$ by

$$
\left(K_{T} f\right)(n)=\sum_{j=1}^{n} k_{T}(j) f_{n-j}=\left(k_{T} * f\right)(n), \quad n \in \mathbb{N} .
$$


By hypothesis, $K_{T}$ is well defined and bounded on $l_{p}(\mathbb{N} ; X)$. By Lemma $2.10,(z-1)^{2} \in$ $\rho(I-T)$ whenever $|z|=1, z \neq 1$. Then, by Proposition 2.7 we have

$$
\begin{aligned}
\hat{k}_{T}(z)=(I-T) \hat{\mathcal{S}}(z) & =z(I-T) R\left((z-1)^{2},(I-T)\right) \\
& =z\left[(z-1)^{2} R\left((z-1)^{2},(I-T)\right)-I\right], \quad z \in \mathbb{T}, z \neq 1 .
\end{aligned}
$$

We observe that there exists $L_{M} \in \mathcal{B}\left(l_{p}(\mathbb{Z} ; X)\right)$ such that

$$
\mathcal{F}\left(L_{M} f\right)(z):=(z-1)^{2} R\left((z-1)^{2},(I-T)\right) \hat{f}(z) .
$$

Explicitly, $L_{M}$ is given by $\left(L_{M} f\right)(n):=\left(K_{T} f\right)(n-1)+f(n)$. We conclude from $[5$, Proposition 1.4] that the set in (ii) is $R$-bounded.

$(i i) \Rightarrow(i)$. Define $M(t)=\left(e^{i t}\right)\left(e^{i t}-1\right)^{2} R\left(\left(e^{i t}-1\right)^{2} ; I-T\right)-\left(e^{i t}\right) I$ for $t \in(0,2 \pi)$. Then $M(t)$ is $R$-bounded by hypothesis and Remark 2.6(b). A calculation show that $M^{\prime}(t)=i e^{i t} N(t)+e^{i t} N^{\prime}(t)$, where $N(t)=\left(e^{i t}-1\right)^{2} R\left(\left(e^{i t}-1\right)^{2} ; I-T\right)-I$. Note that $M(t)$ is $R$-bounded if and only if $N(t)$ is $R$-bounded (cf. Remark 2.6(b)). Moreover

$$
\left(e^{i t}-1\right) N^{\prime}(t)=2 i e^{i t}[N(t)+I]-2 i e^{i t}\left(e^{i t}-1\right)[N(t)+I]^{2} .
$$

It shows that the set $\left\{\left(e^{i t}-1\right) M^{\prime}(t)\right\}_{t \in(0,2 \pi)}$ is $R$-bounded, thanks to Remark 2.6 again. It follows the $R$-boundedness of the set $\left\{\left(e^{i t}+1\right)\left(e^{i t}-1\right) M^{\prime}(t)\right\}$. Then, by Theorem 2.8 we obtain that there exists $T_{M} \in \mathcal{B}\left(l_{p}(\mathbb{Z}, X)\right)$ such that

$$
\mathcal{F}\left(T_{M} f\right)(z)=z(z-1)^{2} R\left((z-1)^{2}, I-T\right) \hat{f}(z)-z \hat{f}(z), \quad z \in \mathbb{T}, z \neq 1 .
$$

By Proposition 2.7, we have $\mathcal{F}\left(\mathcal{C}^{\prime} * f\right)(z)=z(I-T) R\left((z-1)^{2},(I-T)\right) \hat{f}(z)=\mathcal{F}\left(T_{M} f\right)(z)$. Then, by uniqueness of the Fourier transform, we conclude that $\mathcal{K}_{2} \in \mathcal{B}\left(l_{p}(\mathbb{Z}, X)\right)$.

In case of Hilbert spaces, we have the following result.

Corollary 2.12. Let $H$ be a Hilbert space and let $T \in \mathcal{B}(H)$ be power bounded and such that $T$ is analytic. Then the following assertions are equivalent.

(i) $T$ has discrete maximal regularity of order 2.

(ii) $\left\{(\lambda-1)^{2} R\left((\lambda-1)^{2}, I-T\right):|\lambda|=1, \lambda \neq 1\right\}$ is bounded.

\section{REDUCTION TO FIRST ORDER PROBLEMS}

Let $X$ be a Banach space and $T \in \mathcal{B}(X)$. In this section, we consider in $X \times X$ the following problem

$$
v_{n+1}-R_{T} v_{n}=F_{n}
$$

where $v_{n}=\left[x_{n}, \Delta x_{n}\right], F_{n}=\left[0, f_{n}\right]$ and $R_{T} \in \mathcal{B}(X \times X)$ is defined as

$$
R_{T}[x, y]=[x+y, x-T x+y] .
$$

The following is the main result in this section.

Theorem 3.13. Let $T \in \mathcal{B}(X)$ and suppose that $1 \in \rho(T)$. Then $T$ has $l_{p}$-discrete maximal regularity if and only if the operator $R_{T} \in \mathcal{B}(X \times X)$ has $l_{p}$-discrete maximal regularity. 
Proof. Suppose $T$ has $l_{p}$-discrete maximal regularity. Given $F_{n}:=\left[f_{n}, g_{n}\right] \in l_{p}\left(\mathbb{Z}_{+} ; X \times\right.$ $X)$, define

$$
\tilde{G}_{n}:=\left[\tilde{f}_{n}, \tilde{g}_{n}\right]= \begin{cases}{\left[0, g_{0}\right]} & \text { if } n=0 \\ {\left[f_{n}, g_{n}\right]} & \text { if } n \neq 0 .\end{cases}
$$

Let $\tilde{V}_{n}:=\left[\tilde{x}_{n}, \tilde{y}_{n}\right]$ be the solution of the equation

$$
\tilde{V}_{n+1}-R_{T} \tilde{V}_{n}=\tilde{G}_{n}, \quad \tilde{V}_{0}=0,
$$

and let $V_{n}$ the solution of the equation

$$
V_{n+1}-R_{T} V_{n}=F_{n}, \quad V_{0}=0 .
$$

It is easy to see that $V_{n+1}=\tilde{V}_{n+1}+R_{T}^{n}\left(F_{0}-\tilde{G}_{0}\right)$. Then we have only to prove that $\left(\Delta \tilde{V}_{n}\right) \in l_{p}\left(\mathbb{Z}_{+} ; X \times X\right)$.

In fact, according to the definition of $R_{T}$, equation (3.23) is equivalent to the system

which implies that $\tilde{x}_{n}$ must satisfy the equation

$$
\left\{\begin{array}{ccc}
\Delta \tilde{x}_{n}-\tilde{y}_{n} & =\tilde{f}_{n} \\
(T-I) \tilde{x}_{n}+\Delta \tilde{y}_{n} & =\tilde{g}_{n}
\end{array}\right.
$$

$$
\Delta^{2} \tilde{x}_{n}-(I-T) \tilde{x}_{n}=\tilde{h}_{n},
$$

where $\tilde{h}_{n}:=\Delta \tilde{f}_{n}+\tilde{g}_{n}$. Note that $\tilde{x}_{0}=0$ and

$$
\tilde{x}_{1}=\Delta \tilde{x}_{0}+\tilde{x}_{0}=\Delta \tilde{x}_{0}=\tilde{y}_{0}+\tilde{f}_{0}=0 .
$$

Since $\left(\tilde{f}_{n}\right)$ and $\left(\tilde{g}_{n}\right)$ are in $l_{p}\left(\mathbb{Z}_{+} ; X\right)$, we obtain that $\Delta \tilde{f}_{n}=\tilde{f}_{n+1}-\tilde{f}_{n} \in l_{p}\left(\mathbb{Z}_{+} ; X\right)$ and hence $\left(\tilde{h}_{n}\right) \in l_{p}\left(\mathbb{Z}_{+} ; X\right)$. Since $T$ has $l_{p}$-maximal regularity, we get that the solution $\tilde{x}_{n}$ of (3.24) satisfy $\Delta^{2} \tilde{x}_{n} \in l_{p}\left(\mathbb{Z}_{+} ; X\right)$.

Since by hypothesis $I-T$ is invertible, we obtain from (3.24) that $\tilde{x}_{n}$ and then $\Delta \tilde{x}_{n} \in$ $l_{p}\left(\mathbb{Z}_{+} ; X\right)$. Hence $\Delta \tilde{V}_{n}=\left[\Delta \tilde{x}_{n}, \Delta \tilde{y}_{n}\right]=\left[\Delta \tilde{x}_{n}, \Delta^{2} \tilde{x}_{n}-\Delta \tilde{f}_{n}\right] \in l_{p}\left(\mathbb{Z}_{+} ; X \times X\right)$ proving the assertion.

Conversely, suppose $R_{T}$ has $l_{p}$-discrete maximal regularity. Let $\left(f_{n}\right) \in l_{p}\left(\mathbb{Z}_{+} ; X\right)$ be given and let $x_{n}$ be the solution of equation (2.15). Define $F_{n}=\left[0, f_{n}\right]$. By hypothesis, the (unique) solution $v_{n}$ of the equation

$$
\Delta v_{n}-\left(R_{T}-I\right) v_{n}=F_{n}, \quad v_{0}=0
$$

satisfy $\Delta v_{n} \in l_{p}\left(\mathbb{Z}_{+} ; X \times X\right)$. Define $V_{n}=\left[x_{n}, \Delta x_{n}\right]$. Then $V_{0}=\left[x_{0}, \Delta x_{0}\right]=[0,0]$, and

$$
\begin{aligned}
\Delta V_{n}-\left(R_{T}-I\right) V_{n} & =\left[\Delta x_{n}, \Delta^{2} x_{n}\right]-\left(R_{T}-I\right)\left[x_{n}, \Delta x_{n}\right] \\
& =\left[\Delta x_{n}, \Delta^{2} x_{n}\right]-\left[\Delta x_{n}, x_{n}-T x_{n}\right] \\
& =\left[0, \Delta^{2} x_{n}-(I-T) x_{n}\right]=\left[0, f_{n}\right]=F_{n} .
\end{aligned}
$$

We conclude that $\Delta^{2} x_{n} \in l_{p}\left(\mathbb{Z}_{+}, X\right)$, proving the Theorem.

Acknowledgments: This note was originated in a discussion between both authors, while the second author was visiting the Universidade Federal de Pernambuco and after the first author was visiting the Universidad de Santiago de Chile. Both authors are grateful for the hospitality of the respectives institutions. 


\section{REFERENCES}

[1] H. Amann. Quasilinear parabolic functional evolution equations. In: M. Chipot, H. Ninomiya (editors), Recent Avdances in Elliptic and Parabolic Issues. Proc. of the 2004 Swiss - Japanese Seminar, World Scientific, (2006), 19 - 44.

[2] W. Arendt. Semigroups and evolution equations: functional calculus, regularity and kernel estimates. Evolutionary equations. Vol. I, 1-85, Handb. Differ. Equ., North-Holland, Amsterdam, 2004.

[3] W. Arendt, S. Bu, The operator-valued Marcinkiewicz multiplier theorem and maximal regularity. Math. Z. 240, (2002), 311-343.

[4] W. Arendt, S. Bu. Operator-valued Fourier multiplier on periodic Besov spaces and applications. Proc. Edin. Math. Soc. (2) 47 (2004), 15-33.

[5] S. Blunck, Maximal regularity of discrete and continuous time evolution equation. Studia Math. 146 (2), (2001), 157-176.

[6] S. Blunck, Analyticity and discrete maximal regularity of $L_{p}$-spaces. J. Funct. Anal. 183(1), (2001), 211-230.

[7] J. Bourgain, Vector-valued singular integrals and the $H^{1}-B M O$ duality. Probability Theory and Harmonic Analysis, Marcel Dekker, New York, 1986.

[8] Ph. Clément, G. Da Prato, Existence and regularity results for an integral equation with infinite delay in a Banach space. Integral Equations Operator Theory, 11 (1988), 480-500.

[9] Ph. Clément, B. de Pagter, F.A. Sukochev, M. Witvliet, Schauder decomposition and multiplier theorems. Studia Math. 138 (2000), 135-163.

[10] Ph. Clément, S.O. Londen, and G. Simonett. Quasilinear evolutionary equations and continuous interpolation spaces. J. Differential Equations 196 (2) (2004), 418-447.

[11] C. Cuevas, C. Vidal, A note on discrete maximal regularity for functional difference equations with infinite delay. Adv. Difference Equ.( 2006), 1-11.

[12] R. Denk, M. Hieber and J. Prüss. R-boundedness, Fourier multipliers and problems of elliptic and parabolic type. Mem. Amer. Math. Soc. 166 (2003), no. 788.

[13] V. Keyantuo, C. Lizama. Maximal regularity for al class of integro-differential equations with infinite delay in Banach spaces. Studia Math. 168 (1) (2005), 25-49.

[14] V. Keyantuo, C. Lizama. Fourier multipliers and integro-differential equations in Banach spaces. J. London Math. Soc.69 (3) (2004), 737-750.

[15] V. Keyantuo, C. Lizama. Periodic solutions of second order differential equations in Banach spaces. Math. Z. 253(3) (2006), 489514.

[16] C. Palencia, S. Piskarev, On multiplicative perturbations of $C_{0}$-groups and $C_{0}$-cosine operator families. Semigroup Forum. 63, (2001), 127-152.

[17] P. Portal,Discrete time analytic semigroups and the geometry of Banach spaces. Semigroup Forum. 67, (2003), 125-144.

[18] L. Weis, Operator-valued Fourier multiplier theorems and maximal $L_{p}$-regularity. Math. Ann. 319 (2001), 735-758.

[19] L. Weis, A new approach to maximal $L_{p}$-regularity. Lecture Notes Pure Appl. Math. 215, Marcel Dekker, New York, 2001, 195-214.

Universidade Federal de Pernambuco, Departamento de Matemática,

Av. Prof. Luiz Freire, S/N, Recife-PE, CEP. 50540-740, Brazil.

E-mail address: cch@dmat.ufpe.br

Universidad de Santiago de Chile, Departamento de Matemática, Facultad de Ciencias, Casilla 307-Correo 2, Santiago-Chile.

E-mail address: clizama@usach.cl 